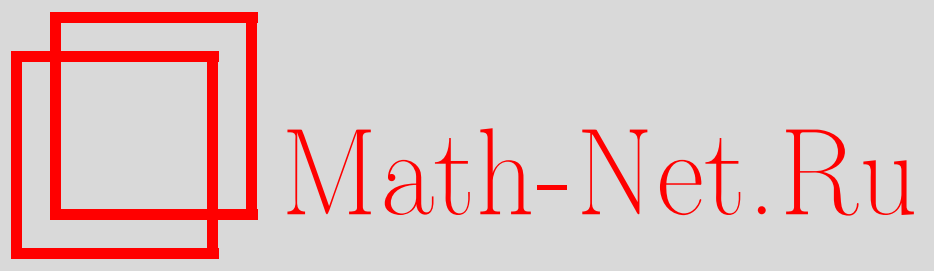

Г. И. Калмыков, Каркасная классификация редуцированных помеченных блоков, Дискрет. матем., 2015, том 27, выпуск 1, 59-72

DOI: https://doi.org/10.4213/dm1315

Использование Общероссийского математического портала Math-Net.Ru подразумевает, что вы прочитали и согласны с пользовательским соглашением http: //www . mathnet.ru/rus/agreement

Параметры загрузки:

IP: 54.210 .77 .194

26 апреля 2023 г., 05:57:56

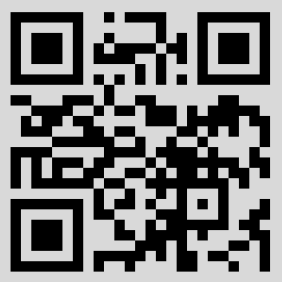




\title{
Каркасная классификация редуцированных помеченных блоков
}

() 2015 г. Г. И. Калмыков*

\begin{abstract}
Описан способ разбиения заданного множества графов на не пересекающиеся подмножества, каждое из которых состоит из совокупности графов, имеющих общий каркас. Указањы множества графов, допускающие такие представления. Отмечена возможность использования этих представлений графов при выводе асимптотических разложений в некоторых задачах статистической механики.
\end{abstract}

Ключевые слова: классификация графов, перечисление графов, каркасные циклы.

Каркасная классификация помеченных графов оказалась полезным математическим инструментом решения некоторых математических проблем в классической статистической механике [1-7]. Не стремясь к максимальной общности, ее можно описать следующим образом. Пусть дано подлежащее классификации множество $\mathfrak{G}(V)$ графов, имеющих одно и то же конечное множество помеченных вершин $V$.

Для данного множества $\mathfrak{G}(V)$ графов с множеством вершин $V$ строится некоторое его подмножество $\widetilde{\mathfrak{G}}(V) \subset \mathfrak{G}(V)$, содержащее (за исключением простейших случаев, когда множество $\mathfrak{G}(V)$ состоит из одного или малого числа графов) значительно меньше графов, чем множество $\mathfrak{G}(V)$. Каждому графу $\widetilde{G} \in \widetilde{G}(V)$ ставится в соответствие множество $X_{\text {ad }}(\widetilde{G})$ ребер. Любое ребро из множества $X_{\text {ad }}(\widetilde{G})$ соединяет две не смежные вершины графа $\widetilde{G}$. Затем каждому графу $\widetilde{G} \in \widetilde{G}(V)$ ставится в соответствие множество $\mathfrak{G}(\widetilde{G})$, состоящее из графа $\widetilde{G}$ и всех его надграфов, которые получаются из графа $\widetilde{G}$ добавлением ребер из множества $X_{\text {ad }}(\widetilde{G})($ см. $[8])$. Если при этом всякий граф из множества $\mathfrak{G}(V)$ входит в одно и только в одно множество вида $\mathfrak{G}(\widetilde{G})$, то в результате получается разбиение множества графов $\mathfrak{G}(V)$ на непересекающиеся подмножества, то есть жлассы, маркируемые графами из множества $\widetilde{\mathfrak{G}}(V)$. А множество графов $\mathfrak{G}(V)$ представляется в виде объединения непересекающихся подмножеств:

$$
\mathfrak{G}(V)=\bigcup_{\widetilde{G} \in \widetilde{\mathfrak{G}}(V)} \widetilde{\mathfrak{G}}(\widetilde{G}) .
$$

При этом граф $\widetilde{G}$, служащий меткой класса графов $\mathfrak{G}(\widetilde{G})$, называется каркасом этих графов, а множество $X_{\text {ad }}(\widetilde{G})$ - множеством допустимых ребер этого каркаса. Классификация такого рода была названа каркасной классификацией [9-12]. В частности, в тех случаях, когда множество каркасов представляет собой множество деревьев, каркасная классификация называется древесной классификацией [12-14].

* Место работы: АНО Центр физических исследований им. П.Н. Лебедева, e-mail: g.i._kalmykov@mail.ru 
Итак, для того, чтобы определить каркасную классификацию данного множества графов, достаточно указать множество каркасов для графов из этого множества, а для каждого из каркасов указать его множество допустимых ребер. В случае удачной каркасной классификации число классов, как, например, в случаях, рассмотренных в [4-6, 9-15], оказывается значительно меньше числа графов в классифицируемом множестве. Иногда в каркасной классификации оказывается более удобным вместо множества каркасов рассматривать другое, находящееся с ним во взаимно однозначном соответствии, множество. Так, в случае каркасной классификации двусвязных помеченных графов (помеченных блоков) в роли такого множества в ряде случаев оказывается удобным рассматривать множество ансамблей каркасных циклов (см. [12] или Приложение).

Решение ряда математических проблем, возникших в теоретической физике (и в частности, в классической статистической механике) упирается в проблему дальнейшего распространения концепции каркасной классификации помеченных графов на новые типы этих графов. В рамках решения проблемы дальнейшего распространения этой концепции в данной статье введено новое понятие редуцированных двусвязных помеченных графов и построена каркасная классификация этих графов. Построение этой классификации является важным вкладом в решение проблемы распространения концепции каркасной классификации помеченных графов на новые типы этих графов.

Далее будут рассматриваться только связные $n$-вершинные помеченные графы без кратных ребер и без петель. Для краткости изложения далее под словом "граф" всюду имеется в виду связный $n$-вершинный помеченный граф без кратных ребер и без петель, а под словом "блок" - двусвязный $n$-вершинный помеченный граф без кратных ребер и без петель. Без ограничения общности можно считать, что вершины этих графов помечены первыми $n$ числами натурального ряда. В дальнейшем изложении каждую вершину такого рода графов будем отождествлять с ее меткой и считать, что множество вершин рассматриваемых графов представляет собой конечное множество $V_{n}=\{1,2, \ldots, n\}$ первых $n$ чисел натурального ряда.

Далее мы всюду будем считать, что множество $V_{n}$ вершин рассматриваемых графов состоит не менее чем из трех натуральных чисел.

Обозначим через $\mathfrak{B}(n ;\{1,2\})$ совокупность всех блоков с множеством вершин $V_{n}=\{1,2, \ldots, n\}$, содержащих ребро $\{1,2\}$.

Определение 1. Граф $\operatorname{Red}(B)$, полученный из блока $B \in \mathfrak{B}(n ;\{1,2\})$ удалением ребра $\{1,2\}$, называется редуцированным блоком.

Пример 1. Рассмотрим граф $C$ с множеством вершин $V_{4}=\{1,2,3,4\}$ и множеством ребер $X(C)=\{\{1,2\},\{1,4\},\{2,3\},\{3,4\}\}$. Граф $C$ является блоком, содержащим ребро $\{1,2\}$ и, следовательно, принадлежащим совокупности блоков $\mathfrak{B}(4 ;\{1,2\})$. Редуцированный блок $\boldsymbol{\operatorname { R e d }}(C)$ представляет собой граф с множеством вершин $V_{4}=\{1,2,3,4\}$ и множеством ребер $X(\operatorname{Red}(C))=\{\{1,4\},\{2,3\},\{3,4\}\}$.

Всюду далее, как, например, в [8], будем отождествлять простой цикл полного графа с помеченными вершинами и помеченный граф, у которого множество вершин совпадает с множеством вершин этого цикла, а множество ребер - с множеством ребер этого цикла, то есть рассматривать такой простой цикл как граф. Будем также отождествлять простую цепь графа с помеченными вершинами и помеченный граф, у которого множество вершин совпадает с множеством вершин этой цепи, а множество ребер - с множеством ребер этой цепи, то есть рассматривать такую 
простую цепь как граф. Простую цепь, соединяющую вершины $v_{0}$ и $v_{h}$, будем обозначать, следуя [8], через $\left(v_{0}-v_{h}\right)$.

Переходим к определению каркасной классификации редуцированных блоков. Это определение опирается на введенное в книге [12] определение каркасной классификации помеченных блоков. Для удобства читателей все необходимые для понимания дальнейшего текста определения, введенные в [12], изложены ниже в разделе "Приложение".

Следуя [12], введем обозначения:

$\mathfrak{B}(n)-$ множество всех двусвязных помеченных графов (блоков) с множеством вершин $V_{n}$;

$\mathfrak{C}(n)=\{\mathbf{C}\}-$ множество ансамблей каркасных циклов (см. [12] или Приложение), где каждый ансамбль удовлетворяет условиям: все вершины циклов этого ансамбля принадлежат множеству вершин $V_{n}$, а каждая вершина из множества $V_{n}$ принадлежит по крайней мере одному из циклов этого ансамбля;

$S(\mathbf{C})$ - объединение всех циклов из ансамбля циклов $\mathbf{C} \in \mathfrak{C}(n)$ (см. [8]);

$\mathfrak{B}(\mathbf{C})$ - множество всех блоков, у которых ансамблем каркасных циклов является ансамбль циклов $\mathbf{C}$.

В [12] доказано, что множество всех блоков с множеством вершин $V$ может быть представлено в виде разложения на непересекающиеся множества (то есть классы), маркируемые ансамблями каркасных циклов (см. [12], или Приложение).

В том весьма важном частном случае, когда множество вершин $V$ совпадает с множеством $V_{n}$, это разложение принимает вид

$$
\mathfrak{B}(n)=\bigcup_{\mathbf{C} \in \mathfrak{C}(n)} \mathfrak{B}(\mathbf{C}) .
$$

Разложение (1) вводит каркасную классификацию помеченных блоков.

Опираясь на эту каркасную классификацию помеченных блоков, введем каркасную классификацию множества $\boldsymbol{\operatorname { R e d }}(\mathfrak{B}(n ;\{1,2\}))=\{\boldsymbol{\operatorname { R e d }}(B): B \in \mathfrak{B}(n ;\{1,2\})\}$ редуцированных блоков.

С этой целью обозначим через $\mathfrak{C}(n ;\{1,2\})$ подмножество множества $\mathfrak{C}(n)$ ансамблей каркасных циклов, состоящее из всех ансамблей множества $\mathfrak{C}(n)$, в которых хотя бы один из циклов содержит ребро $\{1,2\}$. Из определения множества $\mathfrak{C}(n ;\{1,2\})$ и определения объединения циклов $S(\mathbf{C})$ вытекает следствие 1.

Следствие 1. Если ансамбль каркасных ииклов С принадлежит множеству $\mathfrak{C}(n ;\{1,2\})$, то граф $S(\mathbf{C})$ содержит ребро $\{1,2\}$.

Теорема 1. При любом $n \geqslant 3$ множество блоков $\mathfrak{B}(n ;\{1,2\})$ может быть представлено в виде разложения

$$
\mathfrak{B}(n ;\{1,2\})=\bigcup_{\mathbf{C} \in \mathfrak{C}(n ;\{1,2\})} \mathfrak{B}(\mathbf{C})
$$

на непересекающиеся множества, то есть классы.

Доказательство. Пусть $\mathbf{C}$ - ансамбль циклов из множества $\mathfrak{C}(n ;\{1,2\})$. По следствию 1 объединение $S(\mathbf{C})$ всех циклов этого ансамбля также содержит ребро $\{1,2\}$. Значит, и все блоки из класса $\mathfrak{B}(\mathbf{C})$, будучи, по теореме 6.3 из [12], надграфами графа $S(\mathbf{C})$, также содержат ребро $\{1,2\}$. Отсюда следует соотношение 


$$
\mathfrak{B}(n ;\{1,2\}) \supsetneq \bigcup_{\mathbf{C} \in \mathfrak{C}(n ;\{1,2\})} \mathfrak{B}(\mathbf{C}) .
$$

Пусть $B$ - некий блок из сокупности $\mathfrak{B}(n ;\{1,2\})$. Тогда, по определению сокупности $\mathfrak{B}(n ;\{1,2\})$, блок $B$ содержит ребро $\{1,2\}$. Отсюда по замечанию 2.2 из книги [12] следует, что и каркасный цикл $C(0)$ блока $B$ также содержит ребро $\{1,2\}$. Следовательно, ансамбль каркасных циклов этого блока входит во множество $\mathfrak{C}(n ;\{1,2\})$ ансамблей каркасных циклов по определению этого множества, а блок $B$ принадлежит классу блоков, маркируемых ансамблем каркасных циклов, входящим во множество $\mathfrak{C}(n ;\{1,2\})$. Из этих рассуждений следует соотношение

$$
\mathfrak{B}(n ;\{1,2\}) \subsetneq \bigcup_{\mathbf{C} \in \mathfrak{C}(n ;\{1,2\})} \mathfrak{B}(\mathbf{C}) .
$$

Из соотношений 3 и 4 следует утверждение теоремы.

Пример 2. Пусть $n=4$. Найдем разложение множества $\mathfrak{B}(4 ;\{1,2\})$ на непересекающиеся множества, маркируемые ансамблями каркасных циклов.

Множество $\mathfrak{C}(4)$ ансамблей каркасных циклов было найдено в [12] (см. пример 6.12). Три из них содержат по одному циклу. Перечислим эти ансамбли: ансамбль $\mathbf{C}_{1}=\left\{C_{1}(0)\right\}$, где $C_{1}(0)$ - цикл с множеством ребер $X\left(C_{1}(0)\right)=$ $\{\{1,2\},\{1,4\},\{2,3\},\{3,4\}\}$; ансамбль $\mathbf{C}_{2}=\left\{C_{2}(0)\right\}$, где $C_{2}(0)-$ цикл с множеством ребер $X\left(C_{2}(0)\right)=\{\{1,2\},\{1,3\},\{2,4\},\{3,4\}\}$; ансамбль $\mathbf{C}_{3}=\left\{C_{3}(0)\right\}$, где $C_{3}(0)$ - цикл с множеством ребер $X\left(C_{3}(0)\right)=\{\{1,3\},\{1,4\},\{2,3\},\{2,4\}\}$. Остальные два ансамбля каркасных циклов состоят каждый из двух циклов. Это - ансамбль $\mathbf{C}_{4}=\left\{C_{4}(0), C_{4}(0,1)\right\}$, где $C_{4}(0)$ - цикл с множеством ребер $X\left(C_{4}(0)\right)=\{\{1,2\},\{1,3\}\{2,3\}\}$, а $C_{4}(0,1)$ - цикл с множеством ребер $X\left(C_{4}(0,1)\right)=$ $\{\{1,2\},\{1,4\}\{2,4\}\} ;$ и ансамбль $\mathbf{C}_{5}=\left\{C_{5}(0), C_{5}(0,1)\right\}$, где $C_{5}(0)$ - цикл, совпадающий с циклом $C_{4}(0)$, а $C_{5}(0,1)$ - цикл, совпадающий с циклом $C_{2}(0)$.

Из входящих в это множество ансамблей четыре ансамбля, а именно: ансамбли $\mathbf{C}_{1}, \mathbf{C}_{2}, \mathbf{C}_{4}$ и $\mathbf{C}_{5}-$ содержат циклы, обладающие ребром $\{1,2\}$. Следовательно, каркасы $S\left(\mathbf{C}_{1}\right), S\left(\mathbf{C}_{2}\right), S\left(\mathbf{C}_{4}\right)$ и $S\left(\mathbf{C}_{5}\right)$ образуют множество каркасов тех блоков, которые входят в множество $\mathfrak{B}(n ;\{1,2\})$, а ансамбли $\mathbf{C}_{1}, \mathbf{C}_{2}, \mathbf{C}_{4}$ и $\mathbf{C}_{5}$ образуют множество $\mathfrak{C}(4 ;\{1,2\})$. В результате разложение $(2)$ при $n=4$ принимает вид

$$
\mathfrak{B}(4 ;\{1,2\})=\mathfrak{B}\left(\mathbf{C}_{1}\right) \bigcup \mathfrak{B}\left(\mathbf{C}_{2}\right) \bigcup \mathfrak{B}\left(\mathbf{C}_{4}\right) \bigcup \mathfrak{B}\left(\mathbf{C}_{5}\right) .
$$

Лемма 1. Отображение Red множества $\mathfrak{B}(n ;\{1,2\})$ на множество графов $\operatorname{Red}(\mathfrak{B}(n ;\{1,2\}))$ является взаимно однозначным.

Доказательство. Из определения отображения Red следует, что оно является однозначным. Докажем, что, каковы бы ни были два различных блока $B_{1}$ и $B_{2}$ из множества $\mathfrak{B}(n ;\{1,2\})$, их образы $\boldsymbol{\operatorname { R e d }}\left(B_{1}\right)$ и $\boldsymbol{\operatorname { R e d }}\left(B_{2}\right)$ также различны.

Так как оба блока принадлежат множеству $\mathfrak{B}(n ;\{1,2\})$, то они оба содержат ребpo $\{1,2\}$. А так как блоки являются различными и множество вершин одного блока совпадает с множеством вершин другого блока, то множество ребер одного из них не совпадает с множеством ребер другого из этих блоков. Значит, в одном из этих блоков найдется ребро, отличное от ребра $\{1,2\}$ и не принадлежащее другому блоку. Следовательно, множество ребер графа $\boldsymbol{R e d}\left(B_{1}\right)$ не совпадает с множеством ребер графа $\boldsymbol{\operatorname { R e d }}\left(B_{2}\right)$. Стало быть, граф $\boldsymbol{\operatorname { R e d }}\left(B_{1}\right)$ не совпадает с графом $\boldsymbol{\operatorname { R e d }}\left(B_{2}\right)$. Отсюда следует утверждение леммы. 
Так как при любом $\mathbf{C} \in \mathfrak{C}(n ;\{1,2\})$ множество блоков $\mathfrak{B}(\mathbf{C})$ является подмножеством множества блоков $\mathfrak{B}(n ;\{1,2\})$, то из теоремы 1 и леммы 1 вытекает следующее

Следствие 2. Каков бы ни был ансамбль ииклов $\mathbf{C} \in \mathfrak{C}(n ;\{1,2\})$, отображение Red является взаимно однозначным отображением множества $\mathfrak{B}(\mathbf{C})$ на множество $\boldsymbol{\operatorname { R e d }}(\mathfrak{B}(\mathbf{C}))$.

Из теоремы 1, леммы 1 и ее следствия 2 вытекает (см., например, [16]), что множество $\boldsymbol{\operatorname { R e d }}(\mathfrak{B}(n ;\{1,2\}))$ может быть представлено в виде разложения

$$
\boldsymbol{\operatorname { R e d }}(\mathfrak{B}(n ;\{1,2\}))=\bigcup_{\mathbf{C} \in \mathfrak{C}(n ;\{1,2\})} \boldsymbol{\operatorname { R e d }}(\mathfrak{B}(\mathbf{C}))
$$

на непересекающиеся множества, то есть классы.

Пример 3. В примере 2 мы нашли множество $\mathfrak{C}(4 ;\{1,2\})$ ансамблей циклов. Используя этот результат, находим вид, который принимает разложение (6) при $n=4$ :

$$
\boldsymbol{\operatorname { R e d }}(\mathfrak{B}(4 ;\{1,2\}))=\operatorname{Red}\left(\mathfrak{B}\left(\mathbf{C}_{1}\right)\right) \bigcup \boldsymbol{\operatorname { R e d }}\left(\mathfrak{B}\left(\mathbf{C}_{2}\right)\right) \bigcup \boldsymbol{\operatorname { R e d }}\left(\mathfrak{B}\left(\mathbf{C}_{4}\right)\right) \bigcup \boldsymbol{\operatorname { R e d }}\left(\mathfrak{B}\left(\mathbf{C}_{5}\right)\right)
$$

Лемма 2. Каков бы ни был ансамбль ииклов С из множества $\mathfrak{C}(n ;\{1,2\})$, редуиированный блок $\operatorname{Red}(S(\mathbf{C}))$ является подграфом любого графа из множества $\operatorname{Red}(\mathfrak{B}(\mathbf{C}))$.

Доказательство. Каков бы ни был граф $G$ из множества $\operatorname{Red}(\mathfrak{B}(\mathbf{C}))$, в множестве $\mathfrak{B}(\mathbf{C})$ существует блок $B$, являющийся прообразом графа $G$ при отображении Red.

Из определения каркасной классификации помеченных блоков (см. [12] или Приложение) следует, что каркас $S(\mathbf{C})$ является подграфом блока $B$, а множество ребер этого каркаса является подмножеством множества ребер блока В. Поэтому из определения 1 отображения Red следует, что множество ребер редуцированного каркаса $\operatorname{Red}(S(\mathbf{C}))$ является подмножеством множества ребер редуцированного блока $\boldsymbol{\operatorname { R e d }}(B)$, а редуцированный каркас $\boldsymbol{\operatorname { R e d }}(S(\mathbf{C}))$ является подграфом редуцированного блока $G=\operatorname{Red}(B)$.

Теорема 2. Класс редуцированных блоков $\operatorname{Red}(\mathfrak{B}(\mathbf{C}))$, маркируемый ансамблем каркасных ицклов $\mathbf{C} \in \mathfrak{C}(n ;\{1,2\})$, состоит из редуцированного каркаса $\boldsymbol{\operatorname { R e d }}(S(\mathbf{C}))$ и всех графов, получающихся из графа $\operatorname{Red}(S(\mathbf{C}))$ добавлением ребер из множества $X_{\mathrm{ad}}(\mathbf{C})$ допустимых ребер ансамбля каркасных циклов $\mathbf{C}$.

Доказательство. Пусть $B-$ блок из множества $\mathfrak{B}(\mathbf{C})$, где $\mathbf{C} \in \mathfrak{C}(n ;\{1,2\})$. Из определения каркасной классификации помеченных блоков (см. [12] или Приложение) следует, что каркас $S(\mathbf{C})$ является подграфом блока $B$, а множество ребер блока $B$ является объединением множества ребер каркаса $S(\mathbf{C})$ и множества ребер $X_{\mathrm{ad}}(B)$, состоящего из всех тех ребер блока $B$, которые входят в множество $X_{\mathrm{ad}}(\mathbf{C})$ допустимых ребер ансамбля $\mathbf{C}$. Из определения множества $X_{\mathrm{ad}}(\mathbf{C})$ допустимых ребер ансамбля каркасных циклов С (см. [12] или Приложение, определения П.11 и П.14) следует, что всякое допустимое ребро из этого множества не является ребром какого-либо цикла из ансамбля каркасных циклов $\mathbf{C}$ и, в частности, не является ребром $\{1,2\}$. Значит, множество ребер редуцированного блока $\boldsymbol{R e d}(B)$ содержит все ребра из множества $X_{\text {ad }}(B)$. При этом, по лемме 2, редуцированный блок $\boldsymbol{\operatorname { R e d }}(S(\mathbf{C}))$ является подграфом графа $\boldsymbol{\operatorname { R e d }}(B)$ из множества $\boldsymbol{\operatorname { R e d }}(\mathfrak{B}(\mathbf{C}))$. Отсюда следует, что граф $\boldsymbol{\operatorname { R e d }}(B)$ получается добавлением к графу $\boldsymbol{\operatorname { R e d }}(S(\mathbf{C}))$ всех допустимых ребер ансамбля каркасных циклов $\mathbf{C}$, принадлежащих блоку $B$. Итак, 
всякий граф из класса $\boldsymbol{\operatorname { R e d }}(\mathfrak{B}(\mathbf{C}))$ получается из редуцированного блока $\boldsymbol{\operatorname { R e d }}(S(\mathbf{C}))$ добавлением соответствующего набора ребер из множества $X_{\mathrm{ad}}(\mathbf{C})$.

Чтобы закончить доказательство теоремы, осталось показать, что граф $G$, полученный добавлением к редуцированному блоку $\boldsymbol{\operatorname { R e d }}(S(\mathbf{C}))$ любого подмножества $X$ множества допустимых ребер $X_{\mathrm{ad}}(\mathbf{C})$, принадлежит классу $\operatorname{Red}(\mathfrak{B}(\mathbf{C}))$ редуцированных блоков. В самом деле, граф, полученный добавлением к каркасу $S(\mathbf{C})$ множества ребер $X$, является блоком из множества $\mathfrak{B}(\mathbf{C})$. Тогда, как уже доказано выше, редуцированный блок $\boldsymbol{\operatorname { R e d }}(B)$ и есть принадлежащий классу $\boldsymbol{\operatorname { R e d }}(\mathfrak{B}(\mathbf{C}))$ граф, полученный добавлением к редуцированному блоку $\boldsymbol{\operatorname { R e d }}(S(\mathbf{C}))$ множества ребер $X$. Теорема доказана.

Из теоремы 2 вытекает, что каркасная классификация множества $\boldsymbol{\operatorname { R e d }}(\mathfrak{B}(n ;\{1,2\}))$ редуцированных блоков, определенная разложением (6), устроена следующим образом. В этой классификации граф $\boldsymbol{\operatorname { R e d }}(S(\mathbf{C}))$ является каркасом класса редуцированных блоков $\boldsymbol{\operatorname { R e d }}(\mathfrak{B}(\mathbf{C}))$, маркируемого ансамблем каркасных циклов $\mathbf{C}$, а множество ребер $X_{\mathrm{ad}}(\mathbf{C})$, как и в каркасной классификации помеченных блоков, является множеством допустимых ребер класса, маркируемого ансамблем каркасных циклов С.

Пример 4. Найдем множество каркасов тех редуцированных блоков, которые входят в множество $\boldsymbol{\operatorname { R e d }}(\mathfrak{B}(4 ;\{1,2\}))$. В примере 2 было установлено, что блоки $S\left(\mathbf{C}_{1}\right)$, $S\left(\mathbf{C}_{2}\right), S\left(\mathbf{C}_{4}\right)$ и $S\left(\mathbf{C}_{5}\right)$, образуют множество каркасов тех блоков, которые входят в множество $\mathfrak{B}(4 ;\{1,2\})$.

Ансамбли циклов $\mathbf{C}_{1}, \mathbf{C}_{2}, \mathbf{C}_{4}$ и $\mathbf{C}_{5}$ были описаны в примере 2. Так как ансамбли $\mathbf{C}_{1}$ и $\mathbf{C}_{2}$ содержат по одному циклу, то блок $S\left(\mathbf{C}_{1}\right)$ совпадает с циклом $C_{1}(0)$, содержащимся в ансамбле $\mathbf{C}_{1}$, а блок $S\left(\mathbf{C}_{2}\right)$ совпадает с циклом $C_{2}(0)$, содержащимся в ансамбле $\mathbf{C}_{2}$. Отсюда следует, что редуцированный блок $\boldsymbol{R e d}\left(S\left(\mathbf{C}_{1}\right)\right)$ совпадает с редуцированным циклом $\boldsymbol{\operatorname { R e d }}\left(C_{1}(0)\right)$, а редуцированный блок $\boldsymbol{\operatorname { R e d }}\left(S\left(\mathbf{C}_{2}\right)\right)$ - с редуцированным циклом $\boldsymbol{\operatorname { R e d }}\left(C_{2}(0)\right)$. Редуцированный цикл $\boldsymbol{\operatorname { R e d }}\left(C_{1}(0)\right)$ был найден в примере 1. Он представляет собой простую цепь с множеством вершин $V_{4}=\{1,2,3,4\}$ и множеством ребер $X\left(\boldsymbol{\operatorname { R e d }}\left(C_{1}(0)\right)\right)=\{\{1,4\},\{2,3\},\{3,4\}\}$. Точно так же находится редуцированный цикл $\boldsymbol{\operatorname { R e d }}\left(C_{2}(0)\right)$. Он представляет собой цепь с множеством вершин $V_{4}=\{1,2,3,4\}$ и множеством ребер $X\left(\operatorname{Red}\left(C_{2}(0)\right)\right)=$ $\{\{1,3\},\{2,4\},\{3,4\}\}$. Из описания ансамблей $\mathbf{C}_{4}$ и $\mathbf{C}_{5}$, данного в примере 2 , вытекает, что блок $S\left(\mathbf{C}_{4}\right)$ является графом с множеством вершин $V_{4}=\{1,2,3,4\}$ и множеством ребер $X\left(S\left(\mathbf{C}_{4}\right)\right)=\{\{1,2\},\{1,3\},\{1,4\},\{2,3\},\{2,4\}\}$, а блок $S\left(\mathbf{C}_{5}\right)$ является графом с множеством вершин $V_{4}=\{1,2,3,4\}$ и множеством ребер $X\left(S\left(\mathbf{C}_{5}\right)\right)=\{\{1,2\},\{1,3\},\{2,3\},\{2,4\},\{3,4\}\}$. Отсюда следует, что редуцированный блок $\boldsymbol{R e d}\left(S\left(\mathbf{C}_{4}\right)\right)$ является графом с множеством вершин $V_{4}=\{1,2,3,4\}$ и множеством ребер $X\left(\boldsymbol{\operatorname { R e d }}\left(S\left(\mathbf{C}_{4}\right)\right)\right)=\{\{1,3\},\{1,4\},\{2,3\},\{2,4\}\} ;$ а редуцированный блок $\boldsymbol{\operatorname { R e d }}\left(S\left(\mathbf{C}_{5}\right)\right)$ является графом с множеством вершин $V_{4}=\{1,2,3,4\}$ и множеством ребер $X\left(\boldsymbol{\operatorname { R e d }}\left(S\left(\mathbf{C}_{5}\right)\right)\right)=\{\{1,3\},\{2,3\},\{2,4\},\{3,4\}\}$.

Редуцированные блоки $\boldsymbol{\operatorname { R e d }}\left(S\left(\mathbf{C}_{1}\right)\right), \boldsymbol{\operatorname { R e d }}\left(S\left(\mathbf{C}_{2}\right)\right), \boldsymbol{\operatorname { R e d }}\left(S\left(\mathbf{C}_{4}\right)\right)$ и $\boldsymbol{\operatorname { R e d }}\left(S\left(\mathbf{C}_{5}\right)\right)$ образуют множество каркасов тех редуцированных блоков, которые входят в множество $\boldsymbol{\operatorname { R e d }}(\mathfrak{B}(4 ;\{1,2\}))$.

Автор считает своим приятным долгом выразить искреннюю благодарность И.И. Иванчику за постановку задачи и указание возможного применения полученной каркасной классификации редуцированных блоков в классической статистической механике для получения свободных от асимптотической катастрофы (см. 
$[7,14])$ представлений коэффициентов разложения парной функции распределения по степеням плотности.

\section{Приложение}

В Приложении приводятся определения используемых в статье понятий ансамбля каркасных циклов и множества допустимых ребер этого ансамбля, а также обозначений, необходимых для введения этих определений.

Во всякой паре вершин из множества $V_{n}$ вершину с меньшим номером (меткой) будем называть менъшей вершиной этой пары, а другую вершину - большей верииной этой пары. Если множество $V^{\prime}$ содержится в множестве вершин $V_{n}$, то вершину из множества $V^{\prime}$ с наименьшим номером (меткой) мы будем называть наименъшей из вершин множества $V^{\prime}$.

Пусть $C-$ простой цикл с вершинами из множества $V_{n}$. Мы полагаем, что число вершин цикла не менее 3.

Введем обозначения: $V(C)$ - множество вершин цикла $C ; X(C)$ - множество ребер цикла $C ; \alpha(C)$ - младшая вершина цикла $C ; \beta_{1}(C)$ - меньшая из двух вершин, смежных с вершиной $\alpha(C)$ в цикле $C ; \beta_{2}(C)$ - другая вершина цикла $C$, смежная с вершиной $\alpha(C)$ в этом цикле; $d(C)$ - длина простого цикла $C ; v_{h}(C)$ - вершина, которая соединяется с вершиной $\alpha(C)$ простой цепью цикла $C$, имеющей длину $h$ и содержащей вершину $\beta_{1}(C)$. Если вершина $w$ принадлежит множеству вершин цикла $C$, то через $P(C ; w)$ обозначим простую цепь цикла $C$, содержащую вершину $\beta_{1}(C)$ и соединяющую вершины $\alpha(C)$ и $w$.

Определение П.1. Вершина $v$ называется следующей по простому циклу $C$ за вершиной $u$, если длина простой цепи $P(C ; v)$ больше длины простой цепи $P(C ; u)$.

Из определения П.1 вытекает следствие П.1.

Следствие П.1. Если вершина $w$ следует по циклу $C$ за вершиной $v$, а вершина $w^{\prime}$ следует по циклу $C$ за вершиной $w$, то вершина $w^{\prime}$ является вершиной, следующей по циклу $C$ за вершиной $v$.

Доказательство. Цепь $P\left(C ; w^{\prime}\right)$ является объединением цепи $P(C ; w)$ и цепи цикла $C$ (см. [8]), соединяющей вершины $w$ и $w^{\prime}$ и не содержащей вершину $v$. Поэтому длина цепи $P\left(C ; w^{\prime}\right)$ равна сумме длин цепей, входящих в это объединение. Значит, длина цепи $P\left(C ; w^{\prime}\right)$ больше длины цепи $P(C ; w)$. Отсюда по определению П.1 следует, что вершина $w^{\prime}$ следует по циклу $C$ за вершиной $v$.

Определение П.2. Вершина $v$ называется непосредственно следующей по простому циклу $C$ за вершиной $u$, если длина простой цепи $P(C ; v)$ на единицу больше длины простой цепи $P(C ; u)$.

Вершину, непосредственно следующую по простому циклу $C$ за вершиной $u$, обозначим $v(C ; u)$. Из определений П.1 и П.2 вытекает следствие П.2.

Следствие П.2. Вершина, непосредственно следующая по циклу $C$ за вершиной $u$, является вершиной, следующей по циклу $C$ за вершиной $u$.

Пример П.1. Рассмотрим простой цикл $C$ с множеством вершин $V(C)=\{1,2,3,4,5,6\}$ и множеством ребер $X(C)=\{\{1,2\},\{1,4\},\{2,5\}$, $\{3,5\},\{3,6\},\{4,6\}\}$. Здесь $\alpha(C)=1, \beta_{1}(C)=2, \beta_{2}(C)=4, d(C)=6, v_{1}(C)=2$, $v_{2}(C)=5, v_{3}(C)=3, v_{4}(C)=6, v_{5}(C)=4$.

3 Дискретная математика, т. 27 № 1 
Цепь $P(C ; 2)$ является ребром $\{1,2\} . \quad$ Цепь $P(C ; 5)$ являет собой цепь с множеством вершин $V(P(C ; 5))=\{1,2,5\}$ и с множеством ребер $X(P(C ; 5))=\{\{1,2\},\{2,5\}\}$. Цепь $P(C ; 3)$ имеет множество вершин $V(P(C ; 3))=\{1,2,3,5\}$ и множество ребер $X(P(C ; 3))=\{\{1,2\},\{2,5\}$, $\{3,5\}\}$. Цепь $P(C ; 6)$ есть цепь с множеством вершин $V(P(C ; 6))=\{1,2,3,5,6\}$ и множеством ребер $X(P(C ; 6))=\{\{1,2\},\{2,5\},\{3,5\},\{3,6\}\} . \quad$ Цепь $P(C ; 4)$ является цепью с множеством вершин $V(P(C ; 4))=\{1,2,3$, $4,5,6\}$ и множеством ребер $X(P(C ; 4))=\{\{1,2\},\{2,5\},\{3,5\},\{3,6\},\{4,6\}\}$.

Вершина 2 непосредственно следует по циклу $C$ за вершиной 1 . Вершина 5 следует по циклу $C$ за вершиной 1 и следует непосредственно за вершиной 2. Вершина 3 следует по циклу $C$ за вершинами 1 и 2 и следует непосредственно за вершиной 5 . Вершина 6 следует по циклу $C$ за вершинами 1,2 и 5 и следует непосредственно за вершиной 3. Вершина 4 следует по циклу $C$ за вершинами $1,2,3$ и 5 и следует непосредственно за вершиной 6 .

Если $C$ и $C^{\prime}$ - два различных простых цикла с вершинами из множества $V$, то возможны всего четыре случая.

Случай 1. Наименьшая из вершин цикла $C$ не совпадает с наименьшей вершиной цикла $C^{\prime}$, т. е. $\alpha(C) \neq \alpha\left(C^{\prime}\right)$.

Случай 2. Наименьшая вершина цикла $C$ совпадает с наименьшей вершиной цикла $C^{\prime}$, т. е. $\alpha(C)=\alpha\left(C^{\prime}\right)$; а вершина $\beta_{1}(C)$ цикла $C$ не совпадает с вершиной $\beta_{1}\left(C^{\prime}\right)$ цикла $C^{\prime}$, т. е. $\beta_{1}(C) \neq \beta_{1}\left(C^{\prime}\right)$.

Случай 3. Наименьшая вершина цикла $C$ совпадает с наименьшей вершиной цикла $C^{\prime}$, т. е. $\alpha(C)=\alpha\left(C^{\prime}\right)$, а вершина $\beta_{1}(C)$ цикла $C$ совпадает с вершиной $\beta_{1}\left(C^{\prime}\right)$ цикла $C^{\prime}$, т. е. $\beta_{1}(C)=\beta_{1}\left(C^{\prime}\right)$. Существует такое натуральное число $h($ $\left.2 \leqslant h<\min \left\{d(C), d\left(C^{\prime}\right)\right\}\right)$, что вершина $v_{h}(C)$ не совпадает с вершиной $v_{h}\left(C^{\prime}\right)$ (т. е. $\left.v_{h}(C) \neq v_{h}\left(C^{\prime}\right)\right)$, тогда как при всех $i=1,2, \ldots, h-1$ вершина $v_{i}(C)$ совпадает с вершиной $v_{i}\left(C^{\prime}\right)$.

Случай 4. Простые циклы $C$ и $C^{\prime}$ имеют различные длины, т. е. $d(C) \neq \neq d\left(C^{\prime}\right)$. Наименьшая вершина цикла $C$ совпадает с наименьшей вершиной цикла $C^{\prime}$, т. е. $\alpha(C)=\alpha\left(C^{\prime}\right)$. При всех натуральных числах $h$, удовлетворяющих условиям $1 \leqslant$ $h<\min \left\{d(C), d\left(C^{\prime}\right)\right\}$, вершина $v_{h}(C)$ совпадает с вершиной $v_{h}\left(C^{\prime}\right)$.

Определение П.3. Простой цикл $C$ предшествует простому циклу $C^{\prime}$ (обозначение $C \prec C^{\prime}$ ) в следующих четырех случаях: 1) если имеет место случай 1 и $\left.\alpha(C)<\alpha\left(C^{\prime}\right) ; 2\right)$ если имеет место случай 2 и $\left.\beta_{1}(C)<\beta_{1}\left(C^{\prime}\right) ; 3\right)$ если имеет место случай 3 и $\left.v_{h}(C)<v_{h}\left(C^{\prime}\right) ; 4\right)$ если имеет место случай 4 и $d(C)>d\left(C^{\prime}\right)$.

Пример П.2. Пусть $C$ - цикл, введенный в рассмотрение в примере П.1. Сравним цикл $C$ с циклом $C_{1}$, имеющим множество вершин $V\left(C_{1}\right)=\{2,3,4,5\}$ и множество ребер $X\left(C_{1}\right)=\{\{2,4\},\{2,5\},\{3,4\},\{3,5\}\}$. Очевидно, что $\alpha\left(C_{1}\right)=2$. Поскольку $\alpha(C)<\alpha\left(C_{1}\right)$, то цикл $C$ предшествует (в смысле определения П.3) циклу $C_{1}$.

Сравним теперь цикл $C$ с циклом $C_{2}$, имеющим множество вершин $V\left(C_{2}\right)=$ $\{1,2,3,4,5\}$ и множество ребер $X\left(C_{2}\right)=\{\{1,3\},\{1,4\},\{2,4\},\{2,5\},\{3,5\}\}$. Очевидно, что в данном случае $\alpha(C)=\alpha\left(C_{2}\right)=1$ и $\beta_{1}\left(C_{2}\right)=3$. Поскольку $\alpha(C)=\alpha\left(C_{2}\right)$ и $\beta_{1}(C)<\beta_{1}\left(C_{2}\right)$, то цикл $C$ предшествует (в смысле определения П.3) циклу $C_{2}$.

Сравним цикл $C$ с циклом $C_{3}$, имеющим множество вершин $V\left(C_{3}\right)=$ $\{1,2,3,4,5,6\}$ и множество ребер $X\left(C_{3}\right)=\{\{1,2\},\{1,3\},\{2,5\},\{4,5\}$, $\{3,6\},\{4,6\}\}$. Очевидно, что в данном случае $\alpha(C)=\alpha\left(C_{3}\right)=1$, 
$\beta_{1}\left(C_{3}\right)=v_{1}\left(C_{3}\right)=\beta_{1}(C)=v_{1}(C)=2, v_{2}\left(C_{3}\right)=v_{2}(C)=5$ и $v_{3}\left(C_{3}\right)=4>v_{3}(C)=3$. Отсюда следует, что цикл $C$ предшествует (в смысле определения П.3) циклу $C_{3}$.

Наконец, сравним цикл $C$ с циклом $C_{4}$, имеющим множество вершин $V\left(C_{4}\right)=$ $\{1,2,3,5,6\}$ и множество ребер $X\left(C_{4}\right)=\{\{1,2\},\{1,6\},\{2,5\},\{3,5\},\{3,6\}\}$. Очевидно, что в данном случае $\alpha(C)=\alpha\left(C_{4}\right)=1, \beta_{1}\left(C_{4}\right)=v_{1}\left(C_{4}\right)=\beta_{1}(C)=v_{1}(C)=2$, $v_{2}\left(C_{4}\right)=v_{2}(C)=5, v_{3}\left(C_{4}\right)=v_{3}(C)=3, v_{4}\left(C_{4}\right)=v_{4}(C)=6$ и $d(C)=6>d\left(C_{4}\right)=5$. Отсюда следует, что цикл $C$ предшествует (в смысле определения П.3) циклу $C_{4}$.

Обозначим через $I$ совокупность, состоящую из числа 0 и всех упорядоченных конечных наборов, в которых первым элементом является число 0, а все остальные элементы являются натуральными числами. Эти конечные наборы из совокупности $I$ будем называть векторами. А упорядоченные элементы вектора из совокупности $I$ будем называть его компонентами. Число 0 будем рассматривать как нулевой одномерный вектор. ДДля краткости обозначений $(q+1)$-мерный вектор $\left(0, i_{1}, i_{2}, \ldots, i_{q}\right)$ из совокупности $I$ будем обозначать $(i)_{q}$, а нулевой одномерный вектор будем обозначать 0.

Определение П.4. Вектор $(i)_{p}$ называется предшествующим в узком смысле вектору $(j)_{q}$ (символически обозначается $\left.(i)_{p} \prec(j)_{q}\right)$, если $p<q$ и выполняются равенства $i_{n}=j_{n}$ при $n=1,2, \ldots, p$. Одномерный вектор $\mathbf{0}$ предшествует любому другому вектору $(i)_{q}$ из совокупности $I$.

Если вектор $(i)_{p}$ предшествует в узком смысле вектору $(j)_{q}$, то вектор $(j)_{q}$ называется следующим в узком смысле за вектором $(i)_{p}$ (символически обозначается $\left.(j)_{q} \succ(i)_{p}\right)$.

Замечание П.1. Нетрудно доказать, что установленное определением П.4 бинарное отношение на совокупности векторов $I$ транзитивно.

Определение П.5. Вектор $(i)_{p}$ называется предшествующим в широком смысле вектору $(j)_{q}$, если эти векторы удовлетворяют одному из следующих двух условий: (1) $p<q$ и при $n=1,2, \ldots, p$ имеют место равенства $i_{n}=j_{n}$; (2) $p=q, i_{p}<j_{p}$ и при $n=1,2, \ldots, p-1$ имеют место равенства $i_{n}=j_{n}$.

Если вектор $(i)_{p}$ предшествует в широком смысле вектору $(j)_{q}$, то вектор $(j)_{q}$ называется следующим в широком смысле за вектором $(i)_{p}$.

Из определений П.4 и П.5 следует: если вектор $(i)_{p}$ предшествует в узком смысле вектору $(j)_{q}$, то вектор $(i)_{p}$ является предшествующим вектору $(j)_{q}$ также и в широком смысле.

Замечание П.2. Нетрудно доказать, что установленное определением П.5 бинарное отношение на совокупности векторов $I$ транзитивно.

В дальнейшем для краткости мы будем говорить, что вектор $(i)_{p}$ предшествует вектору $(j)_{q}$, если вектор $(i)_{p}$ является предшествующим в узком смысле вектору $(j)_{q}$. Мы также будем говорить для краткости, что вектор $(j)_{q}$ следует за вектором $(i)_{p}$, если вектор $(j)_{q}$ является следующим в узком смысле за вектором $(i)_{p}$.

Всюду далее записью $(i)_{p} \preceq(j)_{q}$ мы будем обозначать бинарное отношение между векторами $(i)_{p}$ и $(j)_{q}$, состоящее в том, что вектор $(i)_{p}$ предшествует вектору $(j)_{q}$ или же совпадает с вектором $(j)_{q}$; а записью $(j)_{q} \succeq(i)_{p}$ мы будем обозначать бинарное отношение между векторами $(j)_{q}$ и $(i)_{p}$, состоящее в том, что вектор $(j)_{q}$ следует за вектором $(i)_{p}$ или же совпадает с вектором $(i)_{p}$. 
Каков бы ни был вектор $(i)_{q}$ (где $q \geqslant 1$ ) из совокупности $I$, через $(i)_{q-1}$ будем обозначать предшествующий ему $q$-мерный вектор из совокупности $I$. В частности, $(i)_{0}$ обозначает нулевой одномерный вектор $\mathbf{0}$, предшествующий всем остальным векторам из совокупности $I$. Далее, каков бы ни был вектор $(i)_{q+p}$ из совокупности $I$ (где $p$ - натуральное число), через $(i)_{q}$ будем обозначать предшествующий ему вектор из совокупности $I$, размерность которого равна $q+1$.

Определение П.6. Множество простых циклов полного графа с множеством вершин $V_{n}$ называется набором ииклов, если каждому циклу из этого множества присвоена метка, являющаяся вектором из совокупности $I$.

Цикл, помеченный вектором $(i)_{q} \neq \mathbf{0}$, будем обозначать $C(i)_{q}$. Цикл, помеченный вектором 0, будем обозначать $C(0)$.

Определение П.7. Вектор $(i)_{q}$, которым помечен простой цикл $C(i)_{q}$, называется вектором-меткой этого цикла.

Обозначим через $I(\mathbf{C})$ множество, состоящее из векторов-меток всех циклов из набора циклов $\mathbf{C}=\left\{C(i)_{q}\right\}$.

Дадим теперь определения ансамбля циклов и ансамбля каркасных циклов, сформулированные в [12]. Здесь будут даны несколько упрощенные формулировки, достаточные, однако, для целей данной статьи.

Определение П.8. Набор циклов $\mathbf{C}=\left\{C(i)_{q}\right\}$ называется ансамблем циклов, если этот набор удовлетворяет следующим условиям:

$\left(\mathcal{A}_{1}\right)$ в множестве $I(\mathbf{C})$ существует вектор-метка $\mathbf{0}$, предшествующий всем остальным векторам-меткам из зтого множества;

$\left(\mathcal{A}_{2}\right)$ если вектор $(i)_{q}$ входит в множество $I(\mathbf{C})$, то и все векторы, предшествующие вектору $(i)_{q}$, но следующие за вектором $\mathbf{0}$, также входят в множество $I(\mathbf{C})$;

$\left(\mathcal{A}_{3}\right)$ если $(i)_{q} \in I(\mathbf{C})$ и $(i)_{q} \neq \mathbf{0}$, то цикл $C(i)_{q}$ содержит хотя бы одну вершину, не принадлежащую циклу $C(i)_{q-1}$;

$\left(\mathcal{A}_{4}\right)$ если $(i)_{q},(j)_{q} \in I(\mathbf{C})$ и вектор $(i)_{q}$ предшествует в широком смысле вектору $(j)_{q}$, то цикл $C(i)_{q}$ предшествует циклу $C(j)_{q}$.

Замечание П.3. Очевидно, что условия $\left(\mathcal{A}_{1}\right)-\left(\mathcal{A}_{4}\right)$ не являются ограничительными для набора циклов, состоящего из одного простого цикла $C(0)$. Поэтому любой такой набор является ансамблем циклов.

Пример П.3. Пусть $\mathbf{C}=\{C(0), C(0,1), C(0,2), C(0,2,1), C(0,2,1,1)\}$ - набор из пяти циклов. Цикл $C(0)$ имеет множество вершин

$$
V(C(0))=\{1,2,3,4,5\}
$$

и множество ребер $X(C(0))=\{\{1,2\},\{1,4\},\{2,5\},\{3,4\},\{3,5\}\}$. Цикл $C(0,1)$ имеет множество вершин $V(C(0,1))=\{1,2,3,5,9\}$ и множество ребер $X(C(0,1))=$ $\{\{1,2\},\{1,9\},\{2,5\},\{3,5\},\{3,9\}\}$. Цикл $C(0,2)$ есть цикл с множеством вершин

$$
V(C(0,2))=\{1,2,3,4,5,6,7\}
$$

и множеством ребер $X(C(0,2))=\{\{1,2\},\{1,4\},\{2,5\},\{3,4\},\{3,7\},\{5,6\},\{6,7\}\}$. Цикл $C(0,2,1)$ представляет собой цикл с множеством вершин

$$
V(C(0,2,1))=\{1,2,3,4,7,8,10\}
$$


и множеством ребер $X(C(0,2,1))=\{\{1,2\},\{1,4\},\{2,8\},\{3,4\},\{3,7\},\{7,10\}$, $\{8,10\}\}$. Наконец, цикл $C(0,2,1,1)$ является циклом с множеством вершин $V(C(0,2,1,1))=\{1,2,3,4,7,8,11\}$ и множеством ребер $X(C(0,2,1,1))=$ $\{\{1,2\},\{1,4\},\{2,8\},\{3,4\},\{3,7\},\{7,11\},\{8,11\}\}$.

В рассматриваемом случае множество векторов-меток $I(\mathbf{C})$ состоит из пяти векторов: $I(\mathbf{C})=\{\mathbf{0},(0,1),(0,2),(0,2,1),(0,2,1,1)\}$. Векторы-метки из этого множества удовлетворяют соотношениям

$$
\mathbf{0} \prec(0,1), \quad 0 \prec(0,2) \prec(0,2,1) \prec(0,2,1,1) .
$$

означающим, что вектор-метка 0 предшествует всем остальным векторам-меткам из множества $I(\mathbf{C})$. Таким образом, набор циклов $\mathbf{C}$ удовлетворяет условию $\left(\mathcal{A}_{1}\right)$. Из этих соотношений следует также, что набор циклов $\mathbf{C}$ удовлетворяет условию $\left(\mathcal{A}_{2}\right)$.

Цикл $C(0,1)$, помеченный вектором-меткой $(0,1)$, следующим за одномерным нулевым вектором 0, содержит вершину 9, не принадлежащую циклу $C(0)$. А цикл $C(0,2)$, помеченный вектором-меткой $(0,2)$, следующим за одномерным нулевым вектором 0, содержит вершины 6 и 7, не принадлежащие циклу $C(0)$. Далее, цикл $C(0,2,1)$, помеченный вектором-меткой $(0,2,1)$, следующим за двумерным вектором $(0,2)$, содержит вершины 8 и 10 , не принадлежащие циклу $C(0,2)$. А цикл $C(0,2,1,1)$, помеченный вектором-меткой $(0,2,1,1)$, следующим за трехмерным вектором $(0,2,1)$, содержит вершину 11 , не принадлежащую циклу $C(0,2,1)$. Таким образом, набор циклов $\mathbf{C}$ удовлетворяет условию $\left(\mathcal{A}_{3}\right)$.

Нетрудно убедиться в том, что входящие в данный набор циклы связаны соотношениями

$$
C(0) \prec C(0,1), \quad C(0) \prec C(0,2) \prec C(0,2,1) \prec C(0,2,1,1) .
$$

Заметим, что по определению П.5 вектор-метка $(0,1)$ предшествует в широком смысле вектору-метке $(0,2)$, а по определению П.3 циклы $C(0,1)$ и $C(0,2)$ удовлетворяют соотношению $C(0,1) \prec C(0,2)$. Отсюда и из (П.4) и (П.5) следует, что набор циклов $\mathbf{C}$ удовлетворяет условию $\left(\mathcal{A}_{4}\right)$.

Итак, набор циклов С удовлетворяет условиям $\left(\mathcal{A}_{1}\right),\left(\mathcal{A}_{2}\right),\left(\mathcal{A}_{3}\right)$ и $\left(\mathcal{A}_{4}\right)$ и является (по определению П.8) ансамблем циклов.

Для определения ансамбля каркасных циклов нам потребуется ввести ряд обозначений и определений.

Если ансамбль циклов $\mathbf{C}=\left\{C(i)_{q}\right\}$ содержит цикл $C(i)_{q}$, то через $\mathbf{C}(i)_{q}$ обозначим набор циклов, состоящий из цикла $C(i)_{q}$ и всех циклов ансамбля $\mathbf{C}$, помеченных векторами, следующими за вектором $(i)_{q}$. Через $S(\mathbf{C})$ обозначим объединение всех циклов из ансамбля циклов $\mathbf{C}$ (см. [8]). Через $S\left(\mathbf{C}(i)_{q}\right)$ обозначим объединение всех циклов из набора $\mathbf{C}(i)_{q}$.

Граф, получающийся в результате удаления из графа $G$ множества вершин $V$, обозначим $G-V$.

Пусть вектор-метка $(i)_{q}$ отличен от вектора-метки 0. Тогда граф $S\left(\mathbf{C}(i)_{q}\right)-$ $V\left(C(i)_{q-1}\right)$ определен. Обозначим такой граф через $D\left((i)_{q} ; \mathbf{C}\right)$, т. е.

$$
D\left((i)_{q} ; \mathbf{C}\right)=S\left(\mathbf{C}(i)_{q}\right)-V\left(C(i)_{q-1}\right)
$$

Через $\Gamma\left((i)_{q} ; \mathbf{C}\right)$ обозначим множество вершин цикла $C(i)_{q-1}$, смежных в графе $S\left(\mathbf{C}(i)_{q-1}\right)$ с вершинами графа $D\left((i)_{q} ; \mathbf{C}\right)$, а через $l\left((i)_{q} ; \mathbf{C}\right)-$ мощность множества $\Gamma\left((i)_{q} ; \mathbf{C}\right)$. 
Замечание П.4. Из свойства $\left(\mathcal{A}_{3}\right)$ (см. определение П.8) вытекает, что граф $D\left((i)_{q} ; \mathbf{C}\right)$ содержит хотя бы одну вершину.

Вершины множества $\Gamma\left((i)_{q} ; \mathbf{C}\right)$ обозначим

$$
\gamma\left((i)_{q}, 1 ; \mathbf{C}\right), \gamma\left((i)_{q}, 2 ; \mathbf{C}\right), \ldots, \gamma\left((i)_{q}, l\left((i)_{q} ; \mathbf{C}\right) ; \mathbf{C}\right) .
$$

В случае $\alpha\left(C(i)_{q-1}\right) \notin \Gamma\left((i)_{q} ; \mathbf{C}\right)$ и $l\left((i)_{q} ; \mathbf{C}\right) \geqslant 2$ предполагается, что вершины из множества $\Gamma\left((i)_{q} ; \mathbf{C}\right)$ удовлетворяют условию: при всех $j$ и $l$, удовлетворяющих неравенствам $1 \leqslant j<l \leqslant l\left((i)_{q} ; \mathbf{C}\right)$, вершина $\gamma\left((i)_{q}, j ; \mathbf{C}\right)$ предшествует вершине $\gamma\left((i)_{q}, l ; \mathbf{C}\right)$ по циклу $C(i)_{q-1}$.

В случае же $\alpha\left(C(i)_{q-1}\right) \in \Gamma\left((i)_{q} ; \mathbf{C}\right)$ и $l\left((i)_{q} ; \mathbf{C}\right) \geqslant 2$ предполагается, что $\alpha\left(C(i)_{q-1}\right)=\gamma\left((i)_{q}, l\left((i)_{q} ; \mathbf{C}\right) ; \mathbf{C}\right)$, а вершины из множества $\Gamma\left((i)_{q} ; \mathbf{C}\right)$ удовлетворяют условию: при всех $j$ и $l$, удовлетворяющих неравенствам $1 \leqslant j<l \leqslant l\left((i)_{q} ; \mathbf{C}\right)-1$, вершина $\gamma\left((i)_{q}, j ; \mathbf{C}\right)$ предшествует вершине $\gamma\left((i)_{q}, l ; \mathbf{C}\right)$ по циклу $C(i)_{q-1}$.

В случаях, когда $l\left((i)_{q} ; \mathbf{C}\right) \geqslant 2$, вершину $\gamma\left((i)_{q}, l\left((i)_{q} ; \mathbf{C}\right)-1 ; \mathbf{C}\right)$ для краткости будем обозначать через $\gamma^{\prime}\left((i)_{q} ; \mathbf{C}\right)$, а вершину $\gamma\left((i)_{q}, l\left((i)_{q} ; \mathbf{C}\right) ; \mathbf{C}\right)$ - через $\gamma^{\prime \prime}\left((i)_{q} ; \mathbf{C}\right)$. Если $(i)_{q} \in I(\mathbf{C}),(i)_{q} \neq \mathbf{0}$ и $l\left((i)_{q} ; \mathbf{C}\right) \geqslant 2$, то через $C^{t}\left((i)_{q} ; \mathbf{C}\right)$ обозначим простую цепь цикла $C(i)_{q-1}$, соединяющую вершины $\gamma^{\prime}\left((i)_{q} ; \mathbf{C}\right)$ и $\gamma^{\prime \prime}\left((i)_{q} ; \mathbf{C}\right)$ и содержащую ребро $\left\{\alpha\left(C(i)_{q-1}\right), \beta_{1}\left(C(i)_{q-1}\right)\right\}$.

Из определения графа $D\left((i)_{q} ; \mathbf{C}\right)$ вытекает следующий вывод: если ансамбль циклов $\mathbf{C}$ обладает циклом $C(i)_{q-1}$, не являющимся гамильтоновым циклом графа $S\left(\mathbf{C}(i)_{q-1}\right)$ (см. [8]), то граф $S\left(\mathbf{C}(i)_{q-1}\right)-V\left(C(i)_{q-1}\right)$ может быть представлен в виде объединения графов вида $D\left((i)_{q} ; \mathbf{C}\right)$ :

$$
S\left(\mathbf{C}(i)_{q-1}\right)-V\left(C(i)_{q-1}\right)=\bigcup_{(i)_{q}} D\left((i)_{q} ; \mathbf{C}\right),
$$

где $\bigcup_{(i)_{q}}$ обозначает объединение по всем векторам $(i)_{q} \in I(\mathbf{C})$ размерности $q+1$, следующим за вектором $(i)_{q-1}$.

Определение П.9. Ребро, соединяющее две вершины простого цикла и не принадлежащее этому циклу, называется его хордой.

Определение П.10. Ансамбль циклов $\mathbf{C}$ называется ансамблем каркасных ииклов, если он удовлетворяет следующим семи условиям:

$\left(\mathcal{B}_{1}\right)$ Каждый цикл из ансамбля $\mathbf{C}$ содержит вершину $\alpha(\mathbf{C})=1$, которая предшествует всем остальным вершинам всех циклов из ансамбля $\mathbf{C}$.

$\left(\mathcal{B}_{2}\right)$ Если $(i)_{q} \in I(\mathbf{C})$, и $(i)_{q} \neq \mathbf{0}$, то $l\left((i)_{q} ; \mathbf{C}\right) \geqslant 2$, а цикл $C(i)_{q}$ содержит простую цепь $C^{t}\left((i)_{q} ; \mathbf{C}\right)$ цикла $C(i)_{q-1}$.

$\left(\mathcal{B}_{3}\right)$ Если $(i)_{q} \in I(\mathbf{C})$, и $(i)_{q} \neq \mathbf{0}$, то цикл $C(i)_{q}$ не содержит ни одной вершины и ни одного ребра цикла $C(i)_{q-1}$, не принадлежащих цепи $C^{t}\left((i)_{q} ; \mathbf{C}\right)$.

$\left(\mathcal{B}_{4}\right)$ При любом $(i)_{q} \in I(\mathbf{C})$ граф $S\left(\mathbf{C}(i)_{q}\right)$ не содержит ни одного простого цикла, предшествующего циклу $C(i)_{q}$.

$\left(\mathcal{B}_{5}\right)$ При всех $(i)_{q} \in I(\mathbf{C})$, удовлетворяющих условию $(i)_{q} \neq \mathbf{0}$, граф $D\left((i)_{q} ; \mathbf{C}\right)-$ связный.

$\left(\mathcal{B}_{6}\right)$ Если два различных вектора $(i)_{q}$ и $\left(i^{\prime}\right)_{q}$, принадлежащих множеству $I(\mathbf{C})$, следуют за одним и тем же вектором $(i)_{q-1} \in I(\mathbf{C})$, то графы $D\left((i)_{q} ; \mathbf{C}\right)$ и $D\left(\left(i^{\prime}\right)_{q} ; \mathbf{C}\right)$ не имеют общих вершин.

$\left(\mathcal{B}_{7}\right)$ Если ансамбль циклов $\mathbf{C}$ содержит цикл $C(i)_{q}$, где $(i)_{q} \neq \mathbf{0}$, то граф $S\left(\mathbf{C}(i)_{q}\right)$ не содержит ни одного ребра, являющегося хордой какого-либо цикла, принадлежащего ансамблю $\mathbf{C}$ и помеченного вектором, предшествующим вектору $(i)_{q}$. 
Теорема П.1. Если ансамбль С удовлетворяет условиям $\left(\mathcal{B}_{1}\right)$ и $\left(\mathcal{B}_{2}\right)$, то граф $S(\mathbf{C})-$ блок.

Определение П.11. Хорда $x$ цикла $C(i)_{q} \in \mathbf{C}$ называется исключаемой хордой этого цикла по отношению $к$ ансамблю $\mathbf{C}$, если эта хорда $x$ является ребром хотя бы одного цикла из ансамбля С. В противном случае хорда $x$ называется сохраняемой хордой этого цикла по отношению к ансамблю С.

Определение П.12. Хорда $\left\{v_{h}(C), v_{h+k}(C)\right\}$ (где $0 \leqslant h \leqslant d(C)-3$ и $2 \leqslant k<$ $d(C)-h)$ простого цикла $C$ удовлетворяет условию следования по этому циклу, если $v_{h+1}(C)<v_{h+k}(C)$.

Пусть $\mathbf{C}$ - ансамбль циклов. Если вектор $(i)_{q}$, где $q>0$, входит в множество $I(\mathbf{C})$, а вершина $\gamma^{\prime \prime}\left((i)_{q} ; \mathbf{C}\right)$ не совпадает с вершиной $\alpha(\mathbf{C})=1$, то через $R\left((i(x, \mathbf{C}))_{q(x, \mathbf{C})} ; \mathbf{C}\right)$ обозначим множество, состоящее из вершины $\alpha(\mathbf{C})=1$ и всех вершин, следующих за вершиной $\gamma^{\prime \prime}\left((i)_{q} ; \mathbf{C}\right)$ по циклу $C_{q-1}$.

Если $x$ - хорда какого-либо цикла из ансамбля $\mathbf{C}$, сохраняемая по отношению к

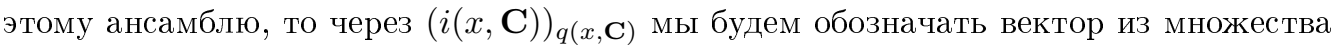
$I(\mathbf{C})$, удовлетворяющий следующему условию.

Условие $\mathcal{D}(x ; \mathbf{C})$. Ребро $x$ является хордой цикла $C(i(x, \mathbf{C}))_{q(x, \mathbf{C})} \in \mathbf{C}$ и не является хордой какого-либо цикла из ансамбля $\mathbf{C}$, помеченного вектором, предшествующим вектору $(i(x, \mathbf{C}))_{q(x, \mathbf{C})}$.

Определение П.13. Ребро $x$ называется запретным ребром ансамбля циклов $\mathbf{C}=$ $\left\{C(i)_{q}\right\}$, если оно по отношению к ансамблю $\mathbf{C}$ является сохраняемой хордой хотя бы одного из циклов этого ансамбля и удовлетворяет следующим условиям:

1) $(i(x, \mathbf{C}))_{q(x, \mathbf{C})} \neq \mathbf{0}$

$2)$ вершина $\alpha(\mathbf{C})$ не содержится в множестве вершин $\Gamma\left((i(x, \mathbf{C}))_{q(x, \mathbf{C})} ; \mathbf{C}\right)$;

$3)$ ребро $x$ соединяет одну из вершин множества $R\left((i(x, \mathbf{C}))_{q(x, \mathbf{C})} ; \mathbf{C}\right)$ с одной из тех вершин цикла $C(i(x, \mathbf{C}))_{q(x, \mathbf{C})}$, которые не принадлежат циклу $C(i(x, \mathbf{C}))_{q(x, \mathbf{C})-1}$.

Определение П.14. Ребро $x$ называется допустимым ребром ансамбля циклов $\mathbf{C}=\left\{C(i)_{q}\right\}$, если оно является по отношению к этому ансамблю сохраняемой хордой хотя бы одного цикла из ансамбля $\mathbf{C}$, удовлетворяет условию следования по циклу $C(i(x, \mathbf{C}))_{q(x, \mathbf{C})}$ и не является запретным ребром этого ансамбля.

Множество всех допустимых ребер ансамбля циклов $\mathbf{C}=\left\{C(i)_{q}\right\}$ обозначим $X_{\mathrm{ad}}(\mathbf{C})$.

\section{Список литературы}

1. Иванчик И. И., Метод ковариантного суммирования диаграмм в класссической статистике, дисс. докт. физ.-матем. наук, Москва, 1987, 273 с.

2. Ivanchik I. I., Generalized Mayer series in classical statistical mechanics, N.Y., Nova Science Publishers, Inc., 1993, 150 c.

3. Иванчик И. И., "Аналитическое представление уравнения состояния в классической статистической механике", Теоретическая и математическая физика, 108:1 (1996), $135-158$.

4. Калмыков Г. И., "О представлении коэффициентов разложения Майера и вириальных коэффициентов", Теоретическал и математическал физика, 84: 2 (1990), 279-289.

5. Калмыков Г. И., "Аналитическое продолжение разложений Майера и вириального разложения", Теоретическая и математическая физика, 92:1 (1992), 139-149. 
6. Калмыков Г. И., "О представлении коэффициентов разложения в степенной ряд плотности распределения одной частицы в большом каноническом ансамбле", Теоретическал и математическал физика, 97:3 (1993), 452-458.

7. Калмыков Г. И., “О явлении асимптотической катастрофы майеровских рядов в классической статистической механике", Теоретическая и математическая физика, 119 №3 (1999), 475-497.

8. Харари Ф., Теория графов, М.: Мир, 1973.

9. Калмыков Г.И., "Каркасная классификация графов", В сб.: Труды IV Междунар. конф. "Дискретные модели в теории управляющих систем" (Красновидово, 2000), ред. В.Б. Алексееев, В.А. Захаров, М.: МАКС Пресс, 2000, 34 с.

10. Калмыков Г. И., "Каркасная классификация помеченных блоков", В сб.: Материаль VII Междунар. сем. "Дискретная математика и ее приложения". Частъ II, ред. О.Б. Лупанов, М.: Изд-во центра прикл. исслед. при механико-матем. фак. МГУ, 2001, 221 с.

11. Калмыков Г. И., "Каркасная классификация помеченных блоков", В сб.: Трудъ V Mеждунар. конф. "Дискретные модели в теории управляющих систем" (Ратмино, 2000), ред. В.Б. Алексееев, А.А. Сапоженко, М.: Изд. отдел фак. вычисл. матем. и кибернетики МГУ, 2003, 39-42.

12. Калмыков Г. И., Каркаснал классификачия помеченных графов, М.: Научный мир, 2006.

13. Калмыков Г. И., Метод древесных сумм и его приложение к решению математических проблем классической статистической механики, дисс. докт. физ.-матем. наук, Москва, 1998, 175 с.

14. Калмыков Г. И., Древесная классификация помеченных графов, М.: Физматлит, 2003.

15. Калмыков Г. И., "О частичном упорядочении деревьев и классификации связных графов и блоков", Дискретная математика, 4:2 (1992), 66-73.

16. Колмогоров А.Н., Фомин С. В., Элементы теории функций и функционалъного анализа, М.: Наука, 1976. 